\title{
Multiple Solutions of $p$-Laplacian with Neumann and Robin Boundary Conditions for Both Resonance and Oscillation Problem
}

\author{
Jing Zhang and Xiaoping Xue \\ Department of Mathematics, Harbin Institute of Technology, Harbin 150025, China \\ Correspondence should be addressed to Jing Zhang, zhangjing127math@yahoo.com.cn \\ Received 29 June 2010; Revised 7 November 2010; Accepted 18 January 2011 \\ Academic Editor: Sandro Salsa
}

Copyright $@ 2011 \mathrm{~J}$. Zhang and X. Xue. This is an open access article distributed under the Creative Commons Attribution License, which permits unrestricted use, distribution, and reproduction in any medium, provided the original work is properly cited.

We discuss Neumann and Robin problems driven by the $p$-Laplacian with jumping nonlinearities. Using sub-sup solution method, Fucík spectrum, mountain pass theorem, degree theorem together with suitable truncation techniques, we show that the Neumann problem has infinitely many nonconstant solutions and the Robin problem has at least four nontrivial solutions. Furthermore, we study oscillating equations with Robin boundary and obtain infinitely many nontrivial solutions.

\section{Introduction}

Let $\Omega$ be a bounded domain of $R^{n}$ with smooth boundary $\partial \Omega$, we consider the following problems:

(i) Neumann problem:

$$
\begin{gathered}
-\Delta_{p} u+\alpha|u|^{p-2} u=f(x, u), \quad \text { in } \Omega, \\
\frac{\partial u}{\partial v}=0, \quad \text { on } \partial \Omega,
\end{gathered}
$$

(ii) Robin problem:

$$
\begin{gathered}
-\Delta_{p} u+\alpha|u|^{p-2} u=f(x, u), \quad \text { in } \Omega, \\
|\nabla u|^{p-2} \frac{\partial u}{\partial v}+b(x)|u|^{p-2} u=0, \quad \text { on } \partial \Omega,
\end{gathered}
$$


where $\Delta_{p} u=\operatorname{div}\left(|\nabla u|^{p-2} \nabla u\right)$ is the $p$-Laplacian operator of $u$ with $1<p<\infty, \alpha>0$, $b(x) \in L^{\infty}(\partial \Omega), b(x) \geq 0$, and $b(x) \neq 0$ on $\partial \Omega, f(x, 0)=0$ for a.e. $x \in \Omega$, and $\partial u / \partial v$ denotes the outer normal derivative of $u$ with respect to $\partial \Omega$. Our purpose is to show the multiplicity of solutions to $\left(p_{1}\right)$ and $\left(p_{2}\right)$.

It is known that $\left(p_{1}\right)$ and $\left(p_{2}\right)$ are the Euler-Lagrange equations of the functionals

$$
\begin{gathered}
J_{1}(u)=\frac{1}{p} \int_{\Omega}|\nabla u|^{p} d x+\frac{\alpha}{p} \int_{\Omega}|u|^{p} d x-\int_{\Omega} F(x, u) d x, \\
J_{2}(u)=\frac{1}{p} \int_{\Omega}|\nabla u|^{p} d x+\frac{\alpha}{p} \int_{\Omega}|u|^{p} d x+\frac{1}{p} \int_{\partial \Omega} b(x)|u|^{p} d s-\int_{\Omega} F(x, u) d x,
\end{gathered}
$$

respectively, defined on the Sobolev space $W^{1, p}(\Omega)$, where $F(x, u)=\int_{0}^{u} f(x, s) d s$. The critical points of functionals correspond to the weak solutions of problems. In Li [1] and Zhang et al. [2], the authors study the existence and multiple solutions of $\left(p_{1}\right)$ and $\left(p_{2}\right)$ using the critical points theory for the semilinear case $p=2$. There also have been some papers dealing with the quasilinear case $p \neq 2$ using the critical point theory, and some existence results of solutions have been generalized to this case in the work of Perera [3], Zhang et al. [4], and Zhang-Li [5]. Most of these papers use the minimax arguments, and nontrivial solutions are obtained with the assumption that the nonlinearity is superlinear at 0 . In this paper, we give the nontrivial solutions of $\left(p_{1}\right)$ and $\left(p_{2}\right)$ with a jumping nonlinearity when the asymptotic limits of the nonlinearity fall in the regions formed by the curves of the Fucík spectrum. Our technique is based on mountain pass theorem, computing the critical groups and Fucík spectrum.

Our general assumptions are the following.

$\left(f_{1}\right)$ There is constant $C>0$ such that $f(x, t)$ satisfies the following subcritical conditions:

$$
|f(x, t)| \leq C\left(|t|^{q}+1\right) \quad \text { for every } x \in \Omega, t \in R,
$$

with $p-1<q<p^{*}-1$, where $p^{*}=n p /(n-p)$ if $n>p$, and $p^{*}=\infty$ if $n=1,2, \ldots, p$.

$\left(f_{2}\right) \exists$ sequence $\left\{a_{i}\right\}$ and $\left\{b_{i}\right\}$, where $a_{i}, b_{i} \in R, i=1,2, \ldots$, which satisfy $a_{i}>0, b_{i}<0$ and $a_{i} \nearrow+\infty, b_{i} \searrow-\infty$ as $i \rightarrow \infty$. And at the same time $\left\{a_{i}\right\},\left\{b_{i}\right\}$ satisfy

$$
f\left(x, a_{i}\right)=\alpha a_{i}^{p-1}, \quad f\left(x, b_{i}\right)=-\alpha\left|b_{i}\right|^{p-1}, \quad \text { for every } x \in \Omega
$$

which means that $\left\{a_{i}\right\},\left\{b_{i}\right\}$ are constant solution sequences of $\left(p_{1}\right)$.

Let $a_{0}=b_{0}=0, f(x, t)<\alpha t^{p-1}$ if $t \in\left(a_{i}, a_{i+1}\right)$, where $i$ is an odd number, $i \geq 1$; $f(x, t)>\alpha t^{p-1}$ if $t \in\left(a_{i}, a_{i+1}\right)$, where $i$ is an even number, $i \geq 0 ; f(x, t)<-\alpha|t|^{p-1}$ if $t \in\left(b_{i+1}, b_{i}\right)$, where $i$ is an even number, $i \geq 0 ; f(x, t)>-\alpha|t|^{p-1}$ if $t \in\left(b_{i+1}, b_{i}\right)$, where $i$ is an odd number, $i \geq 1$, for every $x \in \Omega$.

$\left(f_{3}\right)$ For all $t \neq a_{i}, b_{i}, f$ is $C^{1} ; f_{-}^{\prime}\left(x, a_{i}\right) \neq f_{+}^{\prime}\left(x, a_{i}\right), f_{-}^{\prime}\left(x, b_{i}\right) \neq f_{+}^{\prime}\left(x, b_{i}\right)$, where $i$ is an even number, $i \geq 2, f_{-}^{\prime}(x, t), f_{+}^{\prime}(x, t)$ denote the left and the right derivatives of $f$ at $t$, respectively. 
$\left(f_{4}\right)$ Let $(a, b)=\left(f_{+}^{\prime}\left(x, a_{i}\right)-\alpha, f_{-}^{\prime}\left(x, a_{i}\right)-\alpha\right)$ for $i$ is an even number, $i \geq 2$. For $(a, b) \in R^{2}$, the problem

$$
\begin{gathered}
-\Delta_{p} u=a\left[(u-c)^{+}\right]^{p-1}-b\left[(u-c)^{-}\right]^{p-1}, \quad \text { in } \Omega, \\
\frac{\partial u}{\partial v}=0, \quad \text { on } \partial \Omega,
\end{gathered}
$$

only has constant solution $c$, where $(u-c)^{ \pm}(x)=\max \{ \pm(u-c), 0\}$ and $c$ is a constant.

And $f_{i-}^{\prime}\left(x, a_{i}\right)-\alpha>\lambda_{2}, f_{i+}^{\prime}\left(x, a_{i}\right)-\alpha>\lambda_{2}$ for $i$ is an even number, $i \geq 2$, where

$$
f_{i}(x, t)= \begin{cases}0, & t<0, \\ f(x, t), & 0 \leq t \leq a_{i} \\ f\left(x, a_{i}\right), & t>a_{i}\end{cases}
$$

and $f_{i-}^{\prime}\left(x, a_{i}\right), f_{i+}^{\prime}\left(x, a_{i}\right)$ denote the left and the right derivatives of $f_{i}$ at $a_{i}$, respectively, and $\lambda_{2}$ is the second of the eigenvalue problems with Neumann boundary value condition.

$\left(f_{5}\right) \exists m>\alpha$, such that $f(x, t)+m|t|^{p-2} t$ is increasing in $t$.

In particular, from $\left(f_{2}\right)$, we know that $\left(p_{1}\right)$ has infinitely many constant solutions, a.e., $\left\{a_{i}\right\},\left\{b_{i}\right\}, i=0,1,2, \ldots$. In this paper, we mainly discuss whether it has many nonconstant solutions and what their locations are.

Then we have the main results of this paper.

Theorem 1.1. Assume that $\left(f_{1}\right)-\left(f_{5}\right)$ hold. Then $\left(p_{1}\right)$ has infinitely many nonconstant solutions. Moreover, if one chooses some order intervals which have two pairs of strict constant sub-sup solutions, then $\left(p_{1}\right)$ has at least two nonconstant solutions in some order intervals.

Furthermore, if we assume that $f_{-}^{\prime}(x, 0) \neq f_{+}^{\prime}(x, 0)$ under the same conditions as in Theorem 1.1, we can have at least one sign-changing solution which is of mountain pass type from the mountain pass theorem in order interval. When we discuss multiple solutions of $\left(p_{1}\right)$, we notice that there may be infinitely many sign-changing solutions under stronger assumptions. In fact, if we give more assumptions, we can obtain infinitely many signchanging solutions.

We assume the following.

(F) $F(x, t)>\left(\left(\lambda_{2}+\alpha+\varepsilon_{0}\right) / p\right) t^{p},|t| \geq M, M$ is large enough, where $\lambda_{2}$ is the second eigenvalue of Neumann problem of $-\Delta_{p}$ and $\varepsilon_{0}>0$.

Corollary 1.2. Under the same conditions as in Theorem 1.1, $(F)$ and $f_{-}^{\prime}(x, 0) \neq f_{+}^{\prime}(x, 0)$, then one can get infinitely many sign-changing solutions for $\left(p_{1}\right)$ which are of mountain pass type or not mountain pass type but with positive local degree.

For the Robin problem, if $\exists M_{1}>0, M_{2}>0$ such that $f\left(x, M_{1}\right)=0, f\left(x,-M_{2}\right)=0$ for a.e. $x \in \Omega$, then we give the following assumptions: 
$\left(g_{1}\right) f \in C^{1}\left(\bar{\Omega} \times R^{1} \backslash\{0\}\right), f_{-}^{\prime}(x, 0) \neq f_{+}^{\prime}(x, 0)$, and $\min \left\{f_{+}^{\prime}(x, 0), f_{-}^{\prime}(x, 0)\right\}>\lambda_{1}+\alpha$ for a.e. $x \in \Omega$, where $f_{-}^{\prime}(x, 0), f_{+}^{\prime}(x, 0)$ denote the left and the right derivatives of $f$ at 0 , respectively, and $\lambda_{1}$ is the first eigenvalue of Robin problem of $-\Delta_{p}$;

$\left(g_{2}\right)$ let $(a, b)=\left(f_{+}^{\prime}(x, 0)-\alpha, f_{-}^{\prime}(x, 0)-\alpha\right)$. For $(a, b) \in R^{2}$, the problem

$$
\begin{gathered}
-\Delta_{p} u=a\left(u^{+}\right)^{p-1}-b\left(u^{-}\right)^{p-1}, \quad \text { in } \Omega, \\
|\nabla u|^{p-2} \frac{\partial u}{\partial \mathcal{v}}+b(x)|u|^{p-2} u=0, \quad \text { on } \partial \Omega,
\end{gathered}
$$

only has trivial solution 0 , where $u^{ \pm}(x)=\max \{ \pm u, 0\}$.

In this case, we have the following.

Theorem 1.3. Assume that $\left(f_{1}\right),\left(f_{5}\right),\left(g_{1}\right),\left(g_{2}\right)$ hold. Then one has at least four nontrivial solutions of problem $\left(p_{2}\right)$.

Furthermore, we give the following stronger assumption:

$\left(F^{\prime}\right) F(x, t)>\left[\left(\lambda_{2}+\alpha+\varepsilon_{0}\right) / p+\widetilde{C}\right] t^{p},|t| \geq M, F(x, u)=\int_{0}^{u} f(x, s) d s, u \in E_{2}$, where $E_{2}=$ $\left\{u \in W^{1, p}(\Omega): u=k \varphi_{1}+t \varphi_{2}\right\}, \tilde{C} \gg\left(C^{2} / 2\right)\|b(x)\|_{L^{\infty}(\partial \Omega)}$. Here $C$ is the imbedding constant of Sobolev Trace Theorem (see [6]), $M$ is large enough, $\varepsilon_{0}$ is small enough, $\lambda_{2}$ is the second of the eigenvalue problems with Robin boundary value condition, and $\varphi_{1}, \varphi_{2}$ are the first and the second eigenfunction, respectively.

Then we have the following.

Corollary 1.4. Assume that $f$ is satisfied as in Theorem 1.3 and $\left(F^{\prime}\right)$, then one can have infinitely many sign-changing solutions for $\left(p_{2}\right)$ which are of mountain pass type or not mountain pass type but with positive local degree.

In the oscillating problems of Robin boundary, a.e., $\left(f_{2}\right)$ holds. We make the following assumption.

$\left(F^{\prime \prime}\right) \int_{\Omega} F\left(x, t \varphi_{1}\right) d x \geq\left(\left(\lambda_{1}+\alpha+\varepsilon_{0}\right) / p+\widetilde{C}\right) t^{p} \int_{\Omega} \varphi_{1}^{p} d x,|t| \geq M$, where $\varphi_{1}$ is the first eigenvalue of the Robin problem and $\int_{\Omega} \varphi_{1}^{p} d x=1$.

Then we have the following.

Theorem 1.5. Assume that $f$ is satisfied as in Theorem 1.3 and $\left(f_{2}\right),\left(F^{\prime \prime}\right)$, one can get infinitely many nontrivial solutions of problem $\left(p_{2}\right)$. Some of them are minimum points; others are mountain pass points.

\section{Preliminaries}

Now we recall the notion of critical groups of an isolated critical point $u$ of a $C^{1}$ functional $J$ briefly. Let $U \subset M$ be an isolated neighborhood of $u$ such that there are no critical points of $J$ in $U \backslash\{u\} ; M$ is a Banach space. The critical groups of $u$ are defined as

$$
C_{q}(J, u)=H_{q}\left(J^{c} \cap U,\left(J^{c} \backslash\{u\}\right) \cap U ; G\right), \quad q=0,1,2, \ldots,
$$


where $c=J(u)$ and $J^{c}=\{u \in M \mid J(u) \leq c\}$ is a level set of $J$ and $H_{q}(X, Y ; G)$ are singular relative homology groups with a Abelian coefficient group $G, Y \subset X, q=0,1,2, \ldots$. They are independent of the choices of $U$, hence are well defined. Use $H^{q}(X ; G)$ to stand for the $q$ th singular cohomology group with an Abelian coefficient group $G$; from now on we denote it by $H^{q}(X)$. Assume that $J \in C^{2}(M, R)$, and a critical point $u$ is called nondegenerate if the Hessian $J^{\prime \prime}(u)$ at this point has a bounded inverse. Let $u$ be a nondegenerate critical point of $J$; we call the dimension of the negative space corresponding to the spectral decomposition of $J^{\prime \prime}(u)$, that is, the dimension of the subspace of negative eigenvectors of $J^{\prime \prime}(u)$, the Morse index of $u$, and denote it by ind $\left(J^{\prime \prime}(u)\right)$. If $C_{1}(J, u) \neq 0$, then we call an isolated critical point $u$ of $J$ as a mountain pass point. For the details, we refer to [7]. of $J$.

We have the following basic facts on the critical groups for an isolated critical point

(a) Let $u$ be is an isolated minimum point of $J$, then $C_{q}(J, u)=\delta_{q 0} G$.

(b) If $J \in C^{2}(M, R)$ and $u$ is a nondegenerate critical point of $J$ with Morse index $j$, then $C_{q}(J, u)=\delta_{q j} G$.

Definition 2.1. If any sequence $\left\{u_{k}\right\} \subset M$ which satisfies $J\left(u_{k}\right) \rightarrow c$ and $J^{\prime}\left(u_{k}\right) \rightarrow 0(k \rightarrow \infty)$ has a convergent subsequence, one says that $J$ satisfies the $(P S)_{c}$ condition. If $J$ satisfies $(P S)_{c}$ condition for all $c \in R$, one says that $J$ satisfies the (PS) condition.

Lemma 2.2 (see [8]). Assume that $\underline{u}$ and $\bar{u}$ are, respectively, lower and upper solutions for the problem

$$
\begin{gathered}
-\Delta_{p} u=g(x, u), \quad \text { in } \Omega, \\
|\nabla u|^{p-2} \frac{\partial u}{\partial v}+b(x)|u|^{p-2} u=0, \quad \text { on } \partial \Omega,
\end{gathered}
$$

with $\underline{u} \leq \bar{u}$ a.e. in $\Omega$, where $g(x, s)$ is a Carathéodory function on $\Omega \times R$ with the property that, for any $s_{0}>0$, there exists a constant $A$ such that $|g(x, s)| \leq A$ for a.e. $x \in \Omega$ and all $s \in\left[-s_{0}, s_{0}\right]$. Consider the associated functional

$$
\Phi(u):=\frac{1}{p} \int_{\Omega}|\nabla u|^{p}-\int_{\Omega} G(x, u),
$$

where $G(x, u):=\int_{0}^{s} g(x, t) d t$ and the interval $M:=\left\{u \in W^{1, P}(\Omega): \underline{u} \leq u \leq \bar{u}\right.$ a.e. in $\left.\Omega\right\}$. Then the infimum of $\Phi$ on $M$ is achieved at some $u$, and such $a u$ is a solution of the above problem.

In what follows, we set $X=W^{1, p}(\Omega)$ which is is uniformly convex $(1<p<\infty)$ and equipped with the norm $\|u\|=\left(\int_{\Omega}|\nabla u|^{p} d x+(m+\alpha) \int_{\Omega}|u|^{p} d x\right)^{1 / p}$. Let $E$ be a Hilbert space and $P_{E} \subset E$ a closed convex cone such that $X$ is densely embedded in $E$. Assume that $P=X \cap P_{E}$, $P$ has nonempty interior $\dot{P}$ and any order interval is bounded. It is well known that (PS) condition implies the compactness of the critical set at each level $c \in R$, on the case of the above condition. Then we assume the following:

$\left(J_{1}\right) J \in C^{2}(E, R)$ and satisfies $(P S)$ condition in $E$ and deformation property in $X$; 
$\left(J_{2}\right) \nabla J=i d-K_{E}$, where $K_{E}: E \rightarrow E$ is compact. $K_{E}(X) \subset X$ and the restriction $K=\left.K_{E}\right|_{X}: X \rightarrow X$ is of class $C^{1}$ and strongly preserving, that is, $u \gg v \Leftrightarrow u-v \in \dot{P}$;

$\left(J_{3}\right) J$ is bounded from below on any order interval in $X$.

Lemma 2.3 (Mountain pass theorem in half-order intervals, sup-solutions case (see [9])). Suppose that $J$ satisfies $\left(J_{1}\right)-\left(J_{3}\right) . v_{1}<v_{2}$ is a pair of strict supersolution of $\nabla J=0 . v_{0}<v_{1}$ is a subsolution of $\nabla J=0$. Suppose that $\left[v_{0}, v_{1}\right]$ and $\left[v_{0}, v_{2}\right]$ are admissible invariant sets for $J$. If $J$ has a local strict minimizer $w$ in $\left[v_{0}, v_{2}\right] \backslash\left[v_{0}, v_{1}\right]$. Then $J$ has mountain pass points $u_{0}$ in $\left[v_{0}, v_{2}\right] \backslash$ $\left[v_{0}, v_{1}\right]$.

Lemma 2.4 (Mountain pass theorem in order intervals (see [10])). Suppose that $J$ satisfies $\left(J_{1}\right)$ $\left(J_{3}\right)$ and $\left\{v_{1}, v_{2}\right\},\left\{\omega_{1}, \omega_{2}\right\}$ are two pairs of strict sub-sup solutions of $\nabla J=0$ in $X$ with $v_{1}<\omega_{2}$, $\left[v_{1}, v_{2}\right] \cap\left[\omega_{1}, \omega_{2}\right]=\emptyset$. Then $J$ has a mountain pass point $u_{0}, u_{0} \in\left[v_{1}, \omega_{2}\right] \backslash\left(\left[v_{1}, v_{2}\right] \cup\left[\omega_{1}, \omega_{2}\right]\right)$. More precisely, let $v_{0}$ be the maximal minimizer of $J$ in $\left[v_{1}, v_{2}\right]$ and $\omega_{0}$ the minimal minimizer of $J$ in $\left[\omega_{1}, \omega_{2}\right]$. Then $v_{0} \ll u_{0} \ll \omega_{0}$. Moreover, $C_{1}\left(J, u_{0}\right)$, the critical group of $J$ at $u_{0}$, is nontrivial.

Remark 2.5. (a) Lemma 2.4 still holds if $J \in C^{1}(E, R), K$ is of class $C^{0}$ (see [10]).

(b) For $X=W^{1, p}(\Omega)$, we define $g_{p}(t):=|t|^{p-2} t$. From assumption $\left(f_{5}\right)$, there exists $m>\alpha$ such that $f(x, u)-\alpha|u|^{p-2} u+m g_{p}(u)$ is strictly increasing in $u$. The assumption is not essential but is assumed for simplicity. If such $m$ does not exist then we can approximate $f$ by a sequence of functions so that $m$ as above exists, and obtain the solutions by passing to limits. For $m>\alpha$, we need the operator

$$
A_{m}: X \longrightarrow X, \quad A_{m}(u)=\left(-\Delta_{p}+m g_{p}(\cdot)\right)^{-1}\left((x, u)+m g_{p}(u)\right)
$$

From [11], we know that $A_{m}$ is compact, that is, it is continuous and maps bounded subsets of $X$ into relatively compact subsets of $X$. Since $-\Delta_{p} u+m g_{p}(u)$ is a positive operator,

$$
K:=\left(-\Delta_{p} u+m g_{p}(u)\right)^{-1}\left(f(x, u)-\alpha|u|^{p-2} u+m g_{p}(u)\right)
$$

is strongly orderpreserving. From the above discussion, we have the mountain pass theorem in order intervals of $J_{1}$ and $J_{2}$.

Next, let us recall some notions and known results on Fucík spectrum.

The Fucík spectrum of $p$-Laplacian on $W^{1, p}(\Omega)$ is defined as the set $\Sigma_{p}$ of those points $(a, b) \in R^{2}$ for which the problem

$$
-\Delta_{p} u=a\left(u^{+}\right)^{p-1}-b\left(u^{-}\right)^{p-1}, \quad u \in W^{1, p}(\Omega)
$$

has nontrivial solutions. Here $u^{ \pm}(x)=\max \{ \pm u, 0\}$. 
For the semilinear case $p=2$, it is known that $\Sigma_{2}$ consists, at least locally, of curves emanating from the points $\left(\lambda_{l}, \lambda_{l}\right)$ where $\left\{\lambda_{l}\right\}_{l \in N}$ are the distinct eigenvalues of $-\Delta$ (see, e.g., [12]). It was shown in Schechter [13] that $\Sigma_{2}$ contains continuous and strictly decreasing curves $C_{l_{1}}, C_{l_{2}}$ through $\left(\lambda_{l}, \lambda_{l}\right)$ such that the points in the square $Q_{l}=\left(\lambda_{l-1}, \lambda_{l+1}\right)^{2}$ that are either below the lower curve $C_{l_{1}}$ or above the upper curve $C_{l_{2}}$ are free of $\Sigma_{2}$, while the points on the curves are in $\Sigma_{2}$ when they do not coincide. The points in the region between the curves may or may not belong to $\Sigma_{2}$.

As shown in Lindqvist [14] that the first eigenvalue $\lambda_{1}$ of $-\Delta_{p}$ is positive, simple and admits a strictly positive eigenfunction $\varphi_{1}$, so $\Sigma_{p}$ contains the two lines $\lambda_{1} \times R$ and $R \times \lambda_{1}$. This generalized notion of spectrum was introduced for the semilinear case $p=2$ in the 1970s by Fucík [12] in connection with jumping nonlinearities. A first nontrivial curve $C_{2}$ in $\Sigma_{p}$ through $\left(\lambda_{2}, \lambda_{2}\right)$ that is continuous, strictly decreasing, and asymptotic to $\lambda_{1} \times R$ and $R \times \lambda_{1}$ at infinity was constructed and variationally characterized by a mountain-pass procedure in Cuesta et al. [15].

Consider the problem

$$
\begin{gathered}
-\Delta_{p} u=a\left[(u-c)^{+}\right]^{p-1}-b\left[(u-c)^{-}\right]^{p-1}, \quad \text { in } \Omega, \\
\frac{\partial u}{\partial v}=0, \quad \text { on } \partial \Omega, \\
-\Delta_{p} u=a\left(u^{+}\right)^{p-1}-b\left(u^{-}\right)^{p-1}, \quad \text { in } \Omega, \\
|\nabla u|^{p-2} \frac{\partial u}{\partial v}+b(x)|u|^{p-2} u=0, \quad \text { on } \partial \Omega,
\end{gathered}
$$

from the variational point of view; solutions of (2.7) and (2.8) are the critical points of the functional

$$
\begin{gathered}
I_{1}(u)=I_{1}(u, a, b)=\int_{\Omega}\left(|\nabla u|^{p}-a\left[(u-c)^{+}\right]^{p}-b\left[(u-c)^{-}\right]^{p}\right) d x, \\
I_{2}(u)=I_{2}(u, a, b)=\int_{\Omega}\left(|\nabla u|^{p}-a\left(u^{+}\right)^{p}-b\left(u^{-}\right)^{p}\right) d x+\frac{1}{p} \int_{\partial \Omega} b(x)|u|^{p} d s,
\end{gathered}
$$

respectively, where $c$ is a constant.

If $(a, b)$ does not belong to $\Sigma_{p}, c$ is the constant solution of (2.7), that is, $c$ is an isolated critical point of $I_{1} ; 0$ is the trivial solution of (2.8), that is, 0 is an isolated critical point of $I_{2}$, then from the definition of critical group, we have the $C_{q}\left(I_{1}, c\right)$ and $C_{q}\left(I_{2}, 0\right)$ defined, $q=$ $0,1,2, \ldots$. Now, we give some results relative to the computation of the critical groups which are the results of Dancer and Perera [16]. Let $C_{11}=\left(\left(-\infty, \lambda_{1}\right] \times \lambda_{1}\right) \cup\left(\lambda_{1} \times\left(-\infty, \lambda_{1}\right)\right)$ and $C_{12}=\left(\lambda_{1} \times\left[\lambda_{1},+\infty\right)\right) \cup\left(\left(\lambda_{1},+\infty\right) \times \lambda_{1}\right)$. 
Lemma 2.6. (i) If $(a, b)$ lies below $C_{11}$, then $C_{q}(I, c)=\delta_{q 0} Z$.

(ii) If $(a, b)$ lies between $C_{11}$ and $C_{12}$, then $C_{q}(I, c)=0$ for all $q$.

(iii) If $(a, b)$ lies between $C_{12}$ and $C_{2}$, then $C_{q}(I, c)=\delta_{q 1} Z$.

(iv) If $(a, b)$ does not belong to $\Sigma_{p}$, but lies above $C_{2}$, then $C_{q}(I, c)=0$ for $q=0,1$.

Denote

$$
I_{s}(u)=\int_{\Omega}|\nabla u|^{p}-s\left|(u-c)^{+}\right|^{p}, \quad u \in X,
$$

and $\tilde{I}_{S}$ is the restriction of $I_{S}$ to the $C^{1}$ manifold

$$
S=\left\{u \in X: \int_{\Omega}|u-c|^{p}=1\right\}
$$

As noted in [16], the critical groups of $I$ are related to the homology groups of sublevel sets of $\tilde{I}_{a-b}$. We have that

$$
\left.I\right|_{S}=\tilde{I}_{a-b}-b,
$$

so the sublevel sets

$$
I^{d}=\{u \in X: I(u) \leq d\}, \quad \widetilde{I_{s}^{d}}=\left\{u \in S: \widetilde{I}_{s} \leq d\right\}
$$

are related by

$$
I^{d} \cap S=\widetilde{I}_{a-b}^{d+b}
$$

Lemma 2.7. If $(a, b)$ does not belong to $\Sigma_{p}$, then

$$
C_{q}(I, c) \cong \begin{cases}\delta_{q 0} Z, & \text { if } \widetilde{I}_{a-b}^{b}=\emptyset \\ \widetilde{H}_{q-1}\left(\widetilde{I}_{a-b}^{b}\right), & \text { otherwise }\end{cases}
$$

where $\widetilde{H}_{q}$ denote reduced homology groups. It also holds with $\widetilde{I}_{a-b}^{b}$ replaced by $\bigcirc_{b}=\{u \in S$ : $\left.\tilde{I}_{a-b}(u)>b\right\}$. 


\section{The Proof of the Main Results}

Let

$$
\begin{gathered}
f_{i}(x, t)= \begin{cases}0, & t<0, \\
f(x, t), & 0 \leq t \leq a_{i}, \quad F_{i}(x, t)=\int_{0}^{t} f_{i}(x, s) d s \\
f\left(x, a_{i}\right), & t>a_{i} .\end{cases} \\
J_{1 i}(u)=\frac{1}{p} \int_{\Omega}|\nabla u|^{p} d x+\frac{\alpha}{p} \int_{\Omega}|u|^{p} d x-\int_{\Omega} F_{i}(x, u) d x .
\end{gathered}
$$

It is well known that critical points of $J_{1 i}$ correspond to weak solutions of the following equation:

$$
\begin{gathered}
-\Delta_{p} u+\alpha|u|^{p-2} u=f_{i}(x, u), \quad \text { in } \Omega, \\
\frac{\partial u}{\partial v}=0, \quad \text { on } \partial \Omega .
\end{gathered}
$$

We have that $f_{i}(x, t) \in C^{0}(R, R)$ and $J_{1 i} \in C^{1}(E, R)$. We can discuss similar case for $b_{i}$.

Next, we give the relation of the solutions of $\left(p^{\prime}\right)$ and the solutions of $\left(p_{1}\right)$, that is, Lemma 3.2 below. In order to prove Lemma 3.2, we firstly give the comparison principle.

Let

$$
\begin{gathered}
L_{p}:=-\Delta_{p}+a(x)|u|^{p-2} u, \\
\lambda_{1, p}(a)=\inf \left\{\int_{\Omega}\left[|\nabla u|^{p}+a(x)|u|^{p}\right] d x, u \in W_{0}^{1, p}(\Omega), \int_{\Omega}|u|^{p} d x=1\right\} .
\end{gathered}
$$

Lemma 3.1 (comparison principle (see [17])). Assume $a \in L^{\infty}(\Omega), \lambda_{1, p}(a)>0$. The $L_{p} u \in$ $L^{\infty}(\Omega)$ with $\left.u\right|_{\partial \Omega} \in C^{1+\alpha}(\partial \Omega)$, and $L_{p} u \leq 0$ with $u \in W^{1, p}(\Omega) \cap L^{\infty}(\Omega)$, then $u \leq 0$.

Lemma 3.2. If $u_{i}(x)$ is a solution of $\left(p^{\prime}\right)$, then $u_{i}(x)$ is also a solution of $\left(p_{1}\right)$ and satisfies $0 \leq$ $u_{i}(x) \leq a_{i}, i=1,2, \ldots$

Proof. Suppose that the conclusion is false. Now, consider the domain $U_{i}=\left\{x \in \Omega \mid u_{i}(x)>\right.$ $\left.a_{i}\right\}$, then we have

$$
\begin{gathered}
-\Delta_{p} u=f_{i}(x, u)-\alpha|u|^{p-2} u \leq 0, \quad \text { in } U_{i}, \\
u=a_{i}, \quad \text { on } \partial U_{i},
\end{gathered}
$$

where $-\Delta_{p} u=f_{i}(x, u)-\alpha|u|^{p-2} u=f\left(x, a_{i}\right)-\alpha|u|^{p-2} u \leq f\left(x, a_{i}\right)-\alpha a_{i}^{p-1}=0, x \in U_{i}$ by the definition of $f_{i}(x, u)$. By the comparison principle, we can conclude that $u_{i}(x) \leq 0$ in $U_{i}$. It is a contradiction, so we have that $U_{i}=\emptyset$, that is, $u_{i}(x) \leq a_{i}$.

Similarly, we consider $V_{i}=\left\{x \in \Omega \mid u_{i}(x)<0\right\}$, by the comparison principle; we also get the contradiction, so we have that $V_{i}=\emptyset$, that is, $u_{i}(x) \geq 0$. From the above discussion, we 
have that $0 \leq u_{i}(x) \leq a_{i}, i=1,2, \ldots$ and $f_{i}(x, u)=f\left(x, u_{i}\right)$, so $u_{i}(x)$ is a solution of $\left(p_{1}\right)$. This completes the proof of the lemma.

Remark 3.3. From the above discussion, by applying Lemma 3.2, we know that solutions of $\left(p^{\prime}\right)$ are also the solutions of $\left(p_{1}\right)$ if we want to prove Theorem 1.1, we only need to prove that $\left(p^{\prime}\right)$ has infinitely many nonconstant solutions under the assumptions as in Theorem 1.1 and $\left(p^{\prime}\right)$ has two nonconstant solutions in every order interval.

Theorem 3.4. There are infinitely many nonconstant solutions of $\left(p^{\prime}\right)$. Moreover, if there exists some order intervals which have two pairs of strict constant sub-sup solutions, then there are at least two nonconstant solutions in these order intervals.

Proof. We treat the case of $a_{i}$; the other case of $b_{i}$ is proved by a similar argument.

If $\left(f_{2}\right)$ holds, then

$$
-\Delta_{p} a_{i}=0=f_{i}\left(x, a_{i}\right)-\alpha a_{i}^{p-1}, \quad \text { for a.e. } x \in \Omega,
$$

so $\left\{a_{i}\right\}$ are all positive constant solutions of $\left(p^{\prime}\right)$. Assuming that $i$ is large enough and $i$ is an even number, we also infer that $\left\{a_{2 k-1}\right\}$ are local minimums, $k=1,2, \ldots, i / 2$. So we get $\underline{u}_{2 k-1}$ and $\bar{u}_{2 k-1}$ a strict subsolution and sup-solution pair for $\left(p^{\prime}\right)$, satisfying $\underline{u}_{2 k-1}<a_{2 k-1}<\bar{u}_{2 k-1}$ for each $k, k=1,2, \ldots, i / 2$.

Now, we study the order interval $\left[\underline{u}_{1}, \bar{u}_{3}\right]$ in $X$ which includes two suborder intervals $\left[\underline{u}_{1}, \bar{u}_{1}\right]$ and $\left[\underline{u}_{3}, \bar{u}_{3}\right], a_{2} \in\left[\underline{u}_{1}, \bar{u}_{3}\right]$.

We infer that $J_{1 i}(u)$ satisfies deformation properties and is bounded from below on $\left[\underline{u}_{1}, \bar{u}_{3}\right]$ and so we get a mountain pass point $u_{1} \in\left[\underline{u}_{1}, \bar{u}_{3}\right] \backslash\left(\left[\underline{u}_{1}, \bar{u}_{1}\right] \cup\left[\underline{u}_{3}, \bar{u}_{3}\right]\right)$ according to mountain pass theorem in order interval, we have that $C_{1}\left(J_{1 i}, u_{1}\right)$ is nontrivial.

From assumption $\left(f_{3}\right)$, we know that the left and the right derivatives of $f_{i}$ at $a_{2}$ are different; we consider the problem

$$
\begin{gathered}
-\Delta_{p} u=f_{i}(x, u)-\alpha|u|^{p-2} u, \quad \text { in } \Omega, \\
\frac{\partial u}{\partial v}=0, \quad \text { on } \partial \Omega,
\end{gathered}
$$

where $f_{i} \in C(\bar{\Omega} \times R)$ and as $u \rightarrow a_{2}$ we have

$$
\begin{aligned}
f_{i}(x, u)-\alpha|u|^{p-2} u= & \left(f_{i+}^{\prime}\left(x, a_{2}\right)-\alpha\right)\left[\left(u-a_{2}\right)^{+}\right]^{p-1} \\
& -\left(f_{i-}^{\prime}\left(x, a_{2}\right)-\alpha\right)\left[\left(u-a_{2}\right)^{-}\right]^{p-1}+\circ\left(\left|u-a_{2}\right|^{p-1}\right) .
\end{aligned}
$$

We take $a=f_{i+}^{\prime}\left(x, a_{2}\right)-\alpha, b=f_{i-}^{\prime}\left(x, a_{2}\right)-\alpha$, then from assumption $\left(f_{4}\right)$ and the definition of $\Sigma_{p}$, we know that $(a, b)$ does not belong to $\Sigma_{p}$. So, we have the following.

(1) If $(a, b)$ does not belong to $\Sigma_{p}$, but lies above $C_{2}$, then

$$
C_{q}\left(J_{1 i}, a_{2}\right)=0 \quad \text { for } q=0,1
$$


by Lemma 2.6(iv). In this case, $C_{1}\left(J_{1 i}, a_{2}\right)=0$, so $C_{q}\left(J_{1 i}, a_{2}\right) \supsetneqq C_{q}\left(J_{1 i}, u_{1}\right)$, and we have $u_{1} \neq a_{2}$.

(2) Denote

$$
J_{a-b}(u)=\int_{\Omega}|\nabla u|^{p}-(a-b)\left|\left(u-a_{2}\right)^{+}\right|^{p}, \quad u \in X,
$$

and $\widetilde{J}_{a-b}$ is the restriction of $J_{a-b}$ to the $C^{1}$ manifold

$$
S=\left\{u \in X: \int_{\Omega}\left|u-a_{2}\right|^{p}=1\right\}
$$

where $a=f_{i+}^{\prime}\left(x, a_{2}\right)-\alpha, b=f_{i-}^{\prime}\left(x, a_{2}\right)-\alpha$ as shown above. then

From $\left(f_{4}\right)$, we know that $(a, b)$ does not belong to $\Sigma_{p}$, and if $\tilde{J}_{a-b}(u)>b$, a.e. $\widetilde{J}_{a-b}^{b}=\emptyset$,

$$
C_{q}\left(J_{1 i}, a_{2}\right)=\delta_{q 0} Z
$$

by Lemma 2.7. In this case, $C_{1}\left(J_{1 i}, a_{2}\right)=0$, so $C_{q}\left(J_{1 i}, a_{2}\right) \supsetneqq C_{q}\left(J_{1 i}, u_{1}\right)$, and we have $u_{1} \neq a_{2}$.

Similarly, applying the mountain pass theorem in order interval to $\left[\underline{u}_{3}, \bar{u}_{5}\right]$ which contain two sub-order intervals $\left[\underline{u}_{3}, \bar{u}_{3}\right]$ and $\left[\underline{u}_{5}, \bar{u}_{5}\right]$, we get a mountain pass point $u_{2}$ and prove that $C_{q}\left(J_{1 i}, a_{4}\right) \supsetneqq C_{q}\left(J_{1 i}, u_{2}\right)$, so $u_{2} \neq a_{4}$ from Lemmas 2.6 and 2.7.

We let the procedure go on. So $i / 2-1$ mountain pass points are available which are nonconstant solutions of $\left(p^{\prime}\right)$, where $i$ is large enough and $i$ is an even number. Then we have infinitely many nonconstant positive solutions of $\left(p^{\prime}\right)$ by the arbitrary of $i$. solutions.

We can discuss the similar case for $b_{i}$ and get infinitely many nonconstant negative

Now, we discuss the solutions in $\left[\underline{u}_{1}, \bar{u}_{3}\right]$ more deeply. Since $u_{1}$ is a mountain pass point, for the Leray-Schauder degree of $i d-K^{i}$, we have the computing formular

$$
\operatorname{deg}\left(i d-K^{i}, B\left(u_{1}, r\right), 0\right)=-1 \text {, }
$$

where $r>0$ is small enough, $K^{i}=\left.\left(-\Delta_{p}+(m+\alpha) g_{p}(\cdot)\right)^{-1} f_{i}^{*}\right|_{X}: X \rightarrow X$ is of class $C^{0}$ and strongly preserving, $f_{i}^{*}(x, u)=f_{i}(x, u)+m g_{p}(u)$ (see Remark 2.5(b)). Then according to Poincaré-Hopf formular for $C^{1}$ case and the computation of $C_{q}\left(J_{1 i}, a_{2}\right)$, we have

$$
\operatorname{index}\left(J_{1 i}, a_{2}\right)=(-1)^{l} \text {. }
$$

Furthermore, for minimum points $a_{1}, a_{3}$,

$$
C_{q}\left(J_{1 i}, a_{1}\right) \cong \delta_{q 0} G, \quad C_{q}\left(J_{1 i}, a_{3}\right) \cong \delta_{q 0} G .
$$


From the additivity of Leray-Schauder degree and Theorem 1.1 in [10], we can get

$$
\begin{aligned}
1= & \operatorname{deg}\left(i d-K^{i},\left[\underline{u}_{1}, \bar{u}_{3}\right], 0\right) \\
= & \operatorname{deg}\left(i d-K^{i},\left[\underline{u}_{1}, \bar{u}_{1}\right], 0\right)+\operatorname{deg}\left(i d-K^{i},\left[\underline{u}_{3}, \bar{u}_{3}\right], 0\right)+\operatorname{deg}\left(i d-K^{i}, B\left(a_{2}, r\right), 0\right) \\
& +\operatorname{deg}\left(i d-K^{i}, B\left(u_{1}, r\right), 0\right) \\
= & 1+1+(-1)^{l}+(-1) .
\end{aligned}
$$

So we have $(-1)^{l}=1$. It is impossible. From the above discussion, we conclude that there must exist another critical point $u_{1}^{*} \in\left[\underline{u}_{1}, \bar{u}_{3}\right]$, which satisfies $u_{1}^{*} \neq u_{1}$ and is nonconstant.

Similarly, we can discuss the order interval $\left[\underline{u}_{3}, \bar{u}_{5}\right]$, and we get another critical point $u_{2}^{*} \neq u_{2}$. We let the procedure go on.

This completes the proof of Theorem 3.4.

Thus, we prove that the conclusion of Theorem 1.1 holds.

The Proof of Corollaries 1.2 and 1.4.

Proof. See Theorem 3.5 of Li [1].

Proof of Theorem 1.3. From the variational point of view, solutions of $\left(p_{2}\right)$ are the critical points of the functional

$$
J_{2}(u)=\frac{1}{p} \int_{\Omega}|\nabla u|^{p} d x+\frac{\alpha}{p} \int_{\Omega}|u|^{p} d x+\frac{1}{p} \int_{\partial \Omega} b(x)|u|^{p} d s-\int_{\Omega} F(x, u) d x,
$$

defined on $X:=W^{1, p}(\Omega)$, where $F(x, u)=\int_{0}^{u} f(x, s) d s$.

We show that $J_{2}$ belongs to $C^{1}(X, R)$. In fact, we set

$$
J_{21}(u)=\frac{1}{p} \int_{\Omega}|\nabla u|^{p} d x+\frac{\alpha}{p} \int_{\Omega}|u|^{p} d x-\int_{\Omega} F(x, u) d x, \quad J_{22}(u)=\frac{1}{p} \int_{\partial \Omega} b(x)|u|^{p} d s .
$$

Under the condition $\left(f_{1}\right)$, it is well known that $J_{21}$ is a $C^{1}$-functional. Next, we consider $J_{22}$. If we let $u, v \in X, 0<|t|<1$,

$$
\begin{aligned}
\frac{\left[J_{22}(u+t v)-J_{22}(u)\right]}{t} & =\int_{\partial \Omega} b(x)|u|^{p-2} u v d s+\sum_{q \geq 2} \frac{C_{p}^{q}}{p} t^{q-1} \int_{\partial \Omega} b(x)|u|^{p-q}|v|^{q} d s \\
& \longrightarrow \int_{\partial \Omega} b(x)|u|^{p-2} u v d s, \quad(t \longrightarrow 0) .
\end{aligned}
$$

So we have that $J_{22}$ has a Gateaux derivative and $\left\langle J_{22}^{\prime}(u), v\right\rangle=\int_{\partial \Omega} b(x)|u|^{p-2} u v d s$. 
Let $u_{n} \rightarrow u$ in $X$; now, by Hölder's and Sobolev's inequalities we can estimate

$$
\begin{aligned}
\left|\left\langle J_{22}^{\prime}\left(u_{n}\right)-J_{22}^{\prime}(u), v\right\rangle\right| & =\left|\int_{\partial \Omega} b(x)\left(\left|u_{n}\right|^{p-2} u_{n}-|u|^{p-2} u\right) v d s\right| \\
& \leq\|b\|_{L^{\infty}(\partial \Omega)} \int_{\partial \Omega}\left|\left(\left|u_{n}\right|^{p-2} u_{n}-|u|^{p-2} u\right) v\right| d s \\
& \leq \begin{cases}c\|b\|_{L^{\infty}(\partial \Omega)} \int_{\partial \Omega}\left(\left|u_{n}\right|+|u|\right)^{p-2}\left|u_{n}-u \| v\right| d s & \text { if } p \geq 2, \\
c\|b\|_{L^{\infty}(\partial \Omega)} \int_{\partial \Omega}\left|u_{n}-u\right|^{p-1}|v| d s & \text { if } p<2,\end{cases} \\
& \leq \begin{cases}c\|b\|_{L^{\infty}(\partial \Omega)}\left\|T\left(u_{n}-u\right)\right\|_{L^{p^{\prime}(\partial \Omega)}}\|T v\|_{L^{p}(\partial \Omega)} & \text { if } p \geq 2, \\
c\|b\|_{L^{\infty}(\partial \Omega)}\left\|T\left(u_{n}-u\right)\right\|_{L^{p}(\partial \Omega)}^{p-1}\|T v\|_{L^{p}(\partial \Omega)} & \text { if } p<2,\end{cases}
\end{aligned}
$$

but, when $p \geq 2, p^{\prime}=p /(p-1) \leq p$, we have $\|u\|_{L^{p^{\prime}}} \leq\|u\|_{L^{p}}$, then we have

$$
\begin{aligned}
\left|\left\langle J_{22}^{\prime}\left(u_{n}\right)-J_{22}^{\prime}(u), v\right\rangle\right| & \begin{cases}c\|b\|_{L^{\infty}(\partial \Omega)}\left\|T\left(u_{n}-u\right)\right\|_{L^{p}(\partial \Omega)}\|T v\|_{L^{p}(\partial \Omega)} & \text { if } p \geq 2, \\
c\|b\|_{L^{\infty}(\partial \Omega)}\left\|T\left(u_{n}-u\right)\right\|_{L^{p}(\partial \Omega)}^{p-1}\|T v\|_{L^{p}(\partial \Omega)} & \text { if } p<2,\end{cases} \\
& \leq \begin{cases}c\|b\|_{L^{\infty}(\partial \Omega)}\left\|u_{n}-u\right\|_{W^{1, p}(\Omega)}\|v\|_{W^{1, p}(\Omega)} & \text { if } p \geq 2, \\
c\|b\|_{L^{\infty}(\partial \Omega)}\left\|u_{n}-u\right\|_{W^{1, p}(\Omega)}^{p-1}\|v\|_{W^{1, p}(\Omega)} & \text { if } p<2,\end{cases}
\end{aligned}
$$

where $1 / p+1 / p^{\prime}=1, T: W^{1, p}(\Omega) \rightarrow L^{p}(\partial \Omega)$ is trace operator, and $\|T u\|_{L^{p}(\partial \Omega)} \leq C\|u\|_{W^{1, p}(\Omega)}$ for all $u \in W^{1, p}(\Omega)$ with the constant $C$ depending on $\Omega$ by Sobolev Trace Theorem (see [6]).

To get (3.18), we have used the following well-known inequalities:

$$
\left.|| u\right|^{p-2} u-|v|^{p-2} v \mid \leq \begin{cases}c(|u|+|v|)^{p-2}|u-v| & \text { if } p \geq 2 \\ c|u-v|^{p-1} & \text { if } p<2\end{cases}
$$

which hold for a convenient $c>0, u, v \in R^{n}$. So

$$
\left\|J_{22}^{\prime}\left(u_{n}\right)-J_{22}^{\prime}(u)\right\| \leq\left\{\begin{array}{ll}
c\|b\|_{L^{\infty}(\partial \Omega)}\left\|u_{n}-u\right\|_{W^{1, p}(\Omega)} & \text { if } p \geq 2, \\
c\|b\|_{L^{\infty}(\partial \Omega)}\left\|u_{n}-u\right\|_{W^{1, p}(\Omega)}^{p-1} & \text { if } p<2 .
\end{array} \longrightarrow 0, \quad(n \longrightarrow \infty)\right.
$$

So $J_{22}^{\prime}(u)$ is continuous and $J_{2} \in C^{1}(X, R)$. 
Consider the truncated functions

$$
\tilde{f}(x, t)= \begin{cases}0, & t \leq-M_{2}, \\ f(x, t), & -M_{2} \leq t \leq M_{1}, \\ 0, & t \geq M_{1}\end{cases}
$$

and the corresponding functional

$$
\widetilde{J}(u)=\frac{1}{p} \int_{\Omega}|\nabla u|^{p} d x+\frac{\alpha}{p} \int_{\Omega}|u|^{p} d x+\frac{1}{p} \int_{\partial \Omega} b(x)|u|^{p} d s-\int_{\Omega} \tilde{F}(x, u) d x,
$$

$\tilde{F}(x, t)=\int_{0}^{t} \tilde{f}(x, s) d s$.

From Perera [18] we have that $\tilde{J}$ satisfies $(P S)$ condition. From the deformation theorem, we know that $\widetilde{J}$ satisfies deformation property when $\widetilde{J}$ satisfies $(P S)$ condition. By a similar discussion as in Theorem 1.1, we only need to discuss the critical points of $\tilde{J}$.

Now, we construct the sub-sup solutions of $\left(p_{2}\right)$. It is easy to see that $M_{1}$ is a constant sup-solution of $\left(p_{2}\right)$ and $-M_{2}$ is a constant subsolution. Moreover, we consider $\varepsilon \varphi_{1}$ for all $\varepsilon>0$ small enough. From [14] we know that $\varphi_{1}(x)>0, x \in \bar{\Omega}$. In fact, with $\underline{u}:=\varepsilon \varphi_{1}$, by $\left(g_{1}\right)$ we have

$$
-\Delta_{p} \underline{u}+\alpha|\underline{u}|^{p-2} \underline{u}-f(x, \underline{u})=\varepsilon^{p-1} \varphi_{1}^{p-1}(x)\left[\left(\lambda_{1}+\alpha\right)-\frac{f\left(x, \varepsilon \varphi_{1}\right)}{\varepsilon^{p-1} \varphi_{1}^{p-1}}\right] \leq 0 .
$$

Furthermore, $\varphi_{1} \in W^{1, p}(\Omega) \cap L^{\infty}(\Omega)$ satisfies $-\Delta_{p} \varphi_{1}=\lambda \varphi_{1}^{p-1}$ in the weak sense, then the regularity theory for the $p$-Laplacian (e.g., [19]) implies $\varphi_{1} \in C^{1, \alpha}(\bar{\Omega})$ for some $\alpha=\alpha(n, p) \in$ $(0,1)$. Moreover $\varphi_{1} \geq 0$. In addition, by the strong maximum principle of [20] and $\varphi_{1} \neq 0$, then $\varphi_{1}>0$ in $\Omega$ and $\partial \varphi_{1} / \partial v<0$ on $\partial \Omega$. So if $b(x)$ is small enough at some point $x_{0} \in \partial \Omega$, we can have $\left.\left[|\nabla \underline{u}|^{p-2}(\partial \underline{u} / \partial v)+b(x)|\underline{u}|^{p-2} \underline{u}\right]\right|_{x_{0}} \leq 0$. From the above discussion, we have a sub-solution of $\left(p_{2}\right)$, a.e., $\varepsilon \varphi_{1}\left(x_{0}\right)$. With $\varepsilon \varphi_{1}:=\varepsilon \varphi_{1}\left(x_{0}\right)$.

By a similar argument we can find that $\left[-M_{2},-\varepsilon \varphi_{1}\right]$ is a pair of strict sub-sup solutions.

Now we study the order interval $\left[-M_{2}, M_{1}\right]$ in $X$ which includes two suborder intervals $\left[-M_{2},-\varepsilon \varphi_{1}\right]$ and $\left[\varepsilon \varphi_{1}, M_{1}\right]$. By Lemma 2.2, there exists weak solutions of $\left(p_{2}\right)$ (relative minimum points) $u_{2}, u_{3}$ in $\left[-M_{2},-\varepsilon \varphi_{1}\right]$ and $\left[\varepsilon \varphi_{1}, M_{1}\right]$, respectively. We can infer that $\widetilde{J}(u)$ is bounded from below on $\left[-M_{2}, M_{1}\right]$, so we get a mountain pass point $u_{1} \in$ $\left[-M_{2}, M_{1}\right] \backslash\left(\left[-M_{2},-\varepsilon \varphi_{1}\right] \cup\left[\varepsilon \varphi_{1}, M_{1}\right]\right)$ according to mountain pass theorem in order interval. From the definition of mountain pass point, we have that $C_{1}\left(\widetilde{J}, u_{1}\right)$ is nontrivial.

From assumption $\left(g_{2}\right)$, we know that the left and the right derivatives of $\tilde{f}$ at 0 are different, we consider the problem

$$
\begin{gathered}
-\Delta_{p} u=\tilde{f}(x, u)-\alpha|u|^{p-2} u, \quad \text { in } \Omega, \\
|\nabla u|^{p-2} \frac{\partial u}{\partial \mathcal{v}}+b(x)|u|^{p-2} u=0, \quad \text { on } \partial \Omega,
\end{gathered}
$$


where $\tilde{f} \in C(\bar{\Omega} \times R)$, and as $u \rightarrow 0$ we have

$$
\tilde{f}(x, u)-\alpha|u|^{p-2} u=\left(\tilde{f}_{+}^{\prime}(x, 0)-\alpha\right)\left(u^{+}\right)^{p-1}-\left(\tilde{f}_{-}^{\prime}(x, 0)-\alpha\right)\left(u^{-}\right)^{p-1}+\circ\left(|u|^{p-1}\right) .
$$

We take $a=\tilde{f}_{+}^{\prime}(x, 0)-\alpha, b=\tilde{f}_{-}^{\prime}(x, 0)-\alpha$; then also from assumption $\left(g_{2}\right)$ and the definition of $\Sigma_{p}$, we know that $(a, b) \notin \Sigma_{p}$.

Then we consider the following cases.

(1) If $(a, b)$ does not belong to $\Sigma_{p}$, but lies above $C_{2}$, then

$$
C_{q}(\tilde{J}, 0)=0 \quad \text { for } q=0,1
$$

by Lemma 2.6(iv). In this case, $C_{1}(\tilde{J}, 0)=0$, so $C_{q}(\tilde{J}, 0) \supsetneqq C_{q}\left(\tilde{J}, u_{1}\right)$, we have $u_{1} \neq 0$.

(2) Denote

$$
J_{a-b}(u)=\int_{\Omega}|\nabla u|^{p}-(a-b)\left(u^{+}\right)^{p}+\int_{\partial \Omega} b(x)|u|^{p} d s, \quad u \in X,
$$

and $\tilde{J}_{a-b}$ is the restriction of $J_{a-b}$ to the $C^{1}$ manifold

$$
S=\left\{u \in X: \int_{\Omega}|u|^{p}=1\right\}
$$

where $a=\tilde{f}_{+}^{\prime}(x, 0)-\alpha, b=\tilde{f}_{-}^{\prime}(x, 0)-\alpha$ as shown above.

From $\left(g_{2}\right)$, we know that $(a, b)$ does not belong to $\Sigma_{p}$, if $\tilde{J}_{a-b}(u)>b$, a.e. $\tilde{J}_{a-b}^{b}=\emptyset$, then

$$
C_{q}(\tilde{J}, 0)=\delta_{q 0} Z
$$

by Lemma 2.7. In this case, $C_{1}(\tilde{J}, 0)=0$, so $C_{q}(\tilde{J}, 0) \not C_{q}\left(\tilde{J}, u_{1}\right)$, and we have $u_{1} \neq 0$.

Now, we discuss the solutions in $\left[-M_{2}, M_{1}\right]$ more deeply. We already have four solutions $0, u_{1}, u_{2}, u_{3}$, where $u_{1}$ is the mountain pass point and $u_{2}, u_{3}$ are the local minimum points of $J_{2}$. For the minimum points $u_{2}, u_{3}$, we have

$$
C_{q}\left(\widetilde{J}, u_{2}\right) \cong \delta_{q 0} G, \quad C_{q}\left(\widetilde{J}, u_{3}\right) \cong \delta_{q 0} G
$$

Since $u_{1}$ is a mountain pass point, for the Leray-Schauder degree of $i d-\tilde{K}$, we have the computing formular

$$
\operatorname{deg}\left(i d-\tilde{K}, B\left(u_{1}, r\right), 0\right)=-1
$$

where $r>0$ is small enough, $\tilde{K}=\left.\left(-\Delta_{p}+(m+\alpha) g_{p}(\cdot)\right)^{-1} f^{*}\right|_{X}: X \rightarrow X$ is of class $C^{0}$ and 
strongly order preserving, $f^{*}(x, u)=f(x, u)+m g_{p}(u)$ (see Remark 2.5(b)). Then according to Poincaré-Hopf formula for $C^{1}$ case and the computation of $C_{q}(\tilde{J}, 0)$, we have

$$
\operatorname{index}(\tilde{J}, 0)=(-1)^{d_{l-1}}
$$

From the additivity of Leray-Schauder degree and Theorem 1.1 in [10], we can get

$$
\begin{aligned}
1= & \operatorname{deg}(i d-\tilde{K},[-M, M], 0) \\
= & \operatorname{deg}\left(i d-\tilde{K},\left[-M,-\varepsilon \varphi_{1}\right], 0\right)+\operatorname{deg}\left(i d-\tilde{K},\left[\varepsilon \varphi_{1}, M\right], 0\right) \\
& +\operatorname{deg}(i d-\tilde{K}, B(0, r), 0)+\operatorname{deg}\left(i d-\tilde{K}, B\left(u_{1}, r\right), 0\right) \\
= & 1+1+(-1)^{d_{l-1}}+(-1) .
\end{aligned}
$$

It is impossible. From the above discussion, we conclude that there must exist another critical point $u^{*} \in\left[-M_{2}, M_{1}\right]$, which satisfies $u^{*} \neq u_{1}$ and is nontrivial.

This completes the proof of Theorem 1.3.

Proof of Theorem 1.5. Consider the truncated function

$$
f_{i}(x, t)= \begin{cases}0, & t<0, \\ f(x, t), & 0 \leq t \leq a_{i} \\ f\left(x, a_{i}\right), & t>a_{i} .\end{cases}
$$

Corresponding functional is

$$
J_{i}(u)=\frac{1}{p} \int_{\Omega}|\nabla u|^{p} d x+\frac{\alpha}{p} \int_{\Omega}|u|^{p} d x+\frac{1}{p} \int_{\partial \Omega} b(x)|u|^{p} d s-\int_{\Omega} F_{i}(x, u) d x,
$$

where $F_{i}(x, u)=\int_{0}^{u} f_{i}(x, s) d \mathrm{~s}, i=1,2, \ldots$.

It is known that the solution of $\left(p_{2}\right)$ is also a solution of the following equation as in the same discussion in Lemma 3.2:

$$
\begin{gathered}
-\Delta_{p} u+\alpha|u|^{p-2} u=f_{i}(x, u), \quad \text { in } \Omega, \\
|\nabla u|^{p-2} \frac{\partial u}{\partial v}+b(x)|u|^{p-2} u=0, \quad \text { on } \partial \Omega .
\end{gathered}
$$


By the standard argument we know that $J_{i}$ satisfies $\left(J_{1}\right)-\left(J_{3}\right)$ and the order intervals consisted by sub-super-solutions are admissible invariant set of $J_{i}$. Taking $v_{0}=-M_{2}, v_{1}=a_{1}>0$, then $J_{i}(u)$ has a minimizer $u_{1} \in\left[v_{0}, v_{1}\right]$. By assumption $\left(F^{\prime \prime}\right)$ there exists a $t_{1}>0$ such that

$$
\begin{aligned}
J_{2}\left(t_{1} \varphi_{1}\right) & =\frac{t_{1}^{p}}{p} \int_{\Omega}\left|\nabla \varphi_{1}\right|^{p} d x+\frac{\alpha}{p} t_{1}^{p} \int_{\Omega} \varphi_{1}^{p} d x+\frac{t_{1}^{p}}{p} \int_{\partial \Omega} b(x) \varphi_{1}^{p} d s-\int_{\Omega} F\left(x, t_{1} \varphi_{1}\right) d x \\
& \leq \frac{\left(\lambda_{1}+\alpha\right) t_{1}^{p}}{p} \int_{\Omega} \varphi_{1}^{p} d x-\frac{\left(\lambda_{1}+\alpha+\varepsilon_{0}+\tilde{C}\right) t_{1}^{p}}{p} \int_{\Omega} \varphi_{1}^{p} d x<J_{2}\left(u_{1}\right) .
\end{aligned}
$$

If we take $v_{2}=a_{n_{1}}>t_{1} \varphi_{1}$, where $n_{1}<i$, then

$$
J_{i}\left(t_{1} \varphi_{1}\right)=J_{2}\left(t_{1} \varphi_{1}\right)<J_{i}\left(u_{1}\right)
$$

which implies that $J_{i}(u)$ has a minimizer $u_{2} \in\left[v_{0}, v_{2}\right] \backslash\left[v_{0}, v_{1}\right]$ such that $J_{i}\left(u_{2}\right)<J_{i}\left(u_{1}\right)$. By Lemma 2.3 we get a mountain pass point $u_{3}$. Moreover, $v_{0}<u_{i}<v_{2}, i=1,2,3$, and $u_{i}$ are positive.

Next, we take $v_{1}=a_{n_{1}}, v_{0}=\varepsilon \varphi_{1}$. Then $J_{i}(u)$ has a minimizer $u_{2} \in\left[v_{0}, v_{1}\right]$. By assumption $\left(F^{\prime \prime}\right)$ there is a $t_{2}>0$ such that

$$
J_{2}\left(t_{2} \varphi_{1}\right)<J_{2}\left(u_{2}\right)
$$

If we take $v_{2}=a_{n_{2}}>t_{2} \varphi_{1}$, where $n_{2}<i$, then

$$
J_{i}\left(t_{2} \varphi_{1}\right)=J_{2}\left(t_{2} \varphi_{1}\right)<J_{i}\left(u_{2}\right)
$$

which implies that $J_{i}(u)$ has a minimizer $u_{4} \in\left[v_{0}, v_{2}\right] \backslash\left[v_{0}, v_{1}\right]$ such that $J_{i}\left(u_{4}\right)<J_{i}\left(u_{2}\right)$. By Lemma 2.3 we get a mountain pass point $u_{5}$. Moreover, $v_{0}<u_{i}<v_{2}, i=1,2,3,4,5$, and $u_{i}$ are all positive. Continue making the procedure we obtain the result.

The proof is complete.

Corollary 3.5. Moreover, $\left(p_{1}\right)$ has infinitely many nonconstant negative energy solutions $\left\{u_{k}\right\}$, which are mountain pass types, if the conditions as in Theorem 1.1 hold and $J_{1}\left(a_{2 k}\right) \rightarrow-\infty$ or $J_{1}\left(b_{2 k}\right) \rightarrow-\infty$ as $k \rightarrow+\infty$.

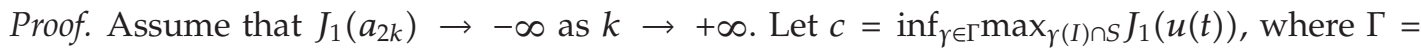
$\left\{\gamma \in C(I, W) \mid \gamma(0)=a_{2 k-1}, \gamma(1)=a_{2 k+1}\right\}$, and $I=[0,1], S=W \backslash\left(W_{1} \cup W_{2}\right), W=\left[\underline{u}_{2 k-1}, \bar{u}_{2 k+1}\right]$, $W_{1}=\left[\underline{u}_{2 k-1}, \bar{u}_{2 k-1}\right], W_{2}=\left[\underline{u}_{2 k+1}, \bar{u}_{2 k+1}\right], c^{*}=J\left(a_{2 k}\right), k=1,2, \ldots$ We discuss the problem in $W$ which have two minimum points $a_{2 k-1}$ and $a_{2 k+1}$. We have that $a_{2 k-1}$ and $a_{2 k+1}$ are in the same radial direction $A=\left\{k e_{1} \mid k \in R\right\}, e_{1}$ is the first eigenvalue function of $\left(-\Delta_{p}+\alpha\right)$ with Neumann boundary. In fact, $e_{1}$ is a constant. We conclude that $c^{*} \geq c$ (see Corollary 3.4 of C. Li and S. Li [21]). Furthermore, if $\left(f_{3}\right),\left(f_{4}\right)$ hold, then $c^{*}>c$. In fact, if $c^{*}=c$, then $c^{*}=\max _{u \in \gamma^{*}(I) \cap S} J(u)=\inf _{\gamma \in \Gamma} \max _{\gamma(I) \cap S} J(u(t))=J\left(a_{2 k}\right)$, where $\gamma^{*}$ is a special path between $a_{2 k-1}$ and $a_{2 k+1}$, which is a path of radial direction $A=\left\{k e_{1} \mid k \in R\right\}$. So $a_{2 k}$ is a mountain pass point. But according to assumptions $\left(f_{3}\right)$ and $\left(f_{4}\right)$, we know that $C_{1}\left(J_{1}, a_{2 k}\right)=0,(l \neq 2)$, that is, $a_{2 k}$ is not a mountain pass type. This is a contradiction. We draw the conclusion. 
Remark 3.6. In Theorems 1.3 and 3.4, we can deal with the case in which $(a, b)$ lies above $C_{2}$, but when $(a, b)$ lies between $C_{12}$ and $C_{2}$, then

$$
\begin{aligned}
& C_{q}\left(J_{1 i}, a_{2}\right) \cong\left\{\begin{array}{ll}
Z, & q=1, \\
0, & q \neq 1,
\end{array} \quad \cong C_{q}\left(J_{1 i}, u_{1}\right),\right. \\
& C_{q}(\tilde{J}, 0) \cong\left\{\begin{array}{ll}
Z, & q=1, \\
0, & q \neq 1,
\end{array} \quad \cong C_{q}\left(\tilde{J}, u_{1}\right),\right.
\end{aligned}
$$

we cannot distinguish $u_{1}$ from $a_{2}$ and 0 , then there may not be nonconstant solutions and nontrivial solutions to $\left(p_{1}\right)$ and $\left(p_{2}\right)$.

Remark 3.7. If we give the assumption

$(\tilde{F}) \int_{\Omega} F(x, u) d x>\left(\left(\mu_{2}+\varepsilon_{0}\right) / 2\right) \int_{\Omega} u^{2} d x$, as $\|u\| \geq M, u \in E_{2}$, where $E_{2}=\{u \in E$ | $\left.u=k_{1} e_{1}+k_{2} e_{2}\right\}, e_{1}, e_{2}$ are the first and the second eigenfunctions of $\left(-\Delta_{p}+\alpha\right\}$ with Neumann boundary, respectively, for all $k_{1}, k_{2} \in R$, $\left\|e_{1}\right\|=\left\|e_{2}\right\|=1, \varepsilon_{0}>0$, and $M$ is large enough,

then under $\left(f_{1}\right)-\left(f_{5}\right)$ and $(\widetilde{F})$, we can obtain infinitely many nonconstant positive, negative, and sign-changing solutions of $\left(p_{1}\right)$.

As a matter of fact, we can infer $(\widetilde{F})$ from $(F)$.

\section{Acknowledgment}

J. Zhang is supported by YJSCX 2008-157 HLJ. X. Xue is supported by NSFC 10971043.

\section{References}

[1] C. Li, "The existence of infinitely many solutions of a class of nonlinear elliptic equations with Neumann boundary condition for both resonance and oscillation problems," Nonlinear Analysis: Theory, Methods \& Applications, vol. 54, no. 3, pp. 431-443, 2003.

[2] J. Zhang, S. Li, Y. Wang, and X. Xue, "Multiple solutions for semilinear elliptic equations with Neumann boundary condition and jumping nonlinearities," Journal of Mathematical Analysis and Applications, vol. 371, no. 2, pp. 682-690, 2010.

[3] K. Perera, "Resonance problems with respect to the Fučík spectrum of the $p$-Laplacian," Electronic Journal of Differential Equations, vol. 2002, no. 36, pp. 1-10, 2002.

[4] Z. Zhang, J. Chen, and S. Li, "Construction of pseudo-gradient vector field and sign-changing multiple solutions involving p-Laplacian," Journal of Differential Equations, vol. 201, no. 2, pp. 287303, 2004.

[5] Z. Zhang and S. Li, "On sign-changing and multiple solutions of the $p$-Laplacian," Journal of Functional Analysis, vol. 197, no. 2, pp. 447-468, 2003.

[6] L. C. Evans, "Partial Differential equations," American Mathematical Society, vol. 19, pp. 327-330, 1998.

[7] K.-C. Chang, Infinite-Dimensional Morse Theory and Multiple Solution Problems, Progress in Nonlinear Differential Equations and Their Applications, 6, Birkhäuser, Boston, Mass, USA, 1993.

[8] D. G. de Figueiredo, J.-P. Gossez, and P. Ubilla, "Local "superlinearity" and "sublinearity" for the p-Laplacian," Journal of Functional Analysis, vol. 257, no. 3, pp. 721-752, 2009.

[9] C. Li, Y. Ding, and S. Li, "Multiple solutions of nonlinear elliptic equations for oscillation problems," Journal of Mathematical Analysis and Applications, vol. 303, no. 2, pp. 477-485, 2005. 
[10] S. Li and Z.-Q. Wang, "Mountain pass theorem in order intervals and multiple solutions for semilinear elliptic Dirichlet problems," Journal d'Analyse Mathématique, vol. 81, pp. 373-396, 2000.

[11] T. Bartsch and Z. Liu, "On a superlinear elliptic $p$-Laplacian equation," Journal of Differential Equations, vol. 198, no. 1, pp. 149-175, 2004.

[12] S. Fučík, "Boundary value problems with jumping nonlinearities," Časopis Pro Pěstování Matematiky, vol. 101, no. 1, pp. 69-87, 1976.

[13] M. Schechter, "The Fučík spectrum," Indiana University Mathematics Journal, vol. 43, no. 4, pp. 1139_ 1157, 1994.

[14] P. Lindqvist, "On the equation $\operatorname{div}\left(|\nabla u|^{p-2} \nabla u\right)+\lambda|u|^{p-2} u=0$," Proceedings of the American Mathematical Society, vol. 109, no. 1, pp. 157-164, 1990.

[15] M. Cuesta, D. de Figueiredo, and J. P. Gossez, "The beginning of the Fučík spectrum for the $p$ Laplacian," Journal of Differential Equations, vol. 159, pp. 212-238, 1999.

[16] N. Dancer and K. Perera, "Some remarks on the Fučík spectrum of the $p$-Laplacian and critical groups," Journal of Mathematical Analysis and Applications, vol. 254, no. 1, pp. 164-177, 2000.

[17] M. X. Wang, "Nonlinear elliptic equations," Manuscript.

[18] K. Perera, “On the Fučík spectrum of the $p$-Laplacian," Nonlinear Differential Equations and Applications, vol. 11, no. 2, pp. 259-270, 2004.

[19] G. M. Lieberman, "Boundary regularity for solutions of degenerate elliptic equations," Nonlinear Analysis: Theory, Methods \& Applications, vol. 12, no. 11, pp. 1203-1219, 1988.

[20] J. L. Vázquez, "A strong maximum principle for some quasilinear elliptic equations," Applied Mathematics and Optimization, vol. 12, no. 3, pp. 191-202, 1984.

[21] C. Li and S. Li, "Multiple solutions and sign-changing solutions of a class of nonlinear elliptic equations with Neumann boundary condition," Journal of Mathematical Analysis and Applications, vol. 298, no. 1, pp. 14-32, 2004. 